\title{
Assignment of (n,m) Raman and Absorption Spectral Features of Metallic Single-Walled Carbon Nanotubes
}

\author{
Michael S. Strano, Erik H. Haroz, Carter Kittrell, Robert H. Hauge, and \\ Richard E. Smalley \\ Department of Chemistry, Center for Nanoscale Science and Technology, and Center for Biological \\ and Environmental Nanotechnology \\ Rice University \\ Houston, Texas 77005 USA
}

\begin{abstract}
The (n,m) spectral features for isolated metallic single walled carbon nanotubes were deduced by examining Raman excitation profiles. Correlation of the radial breathing mode frequency with diameter identifies the $(n, m)$ index of the metallic tube. Observation of the energy of Raman intensity maximum provides experimental values for the optical transitions directly, and allows for model independent estimation of peak splitting and diameter scaling. The results were extrapolated to all metallic nanotubes using a parameterized functional form deduced from the framework of the Tight Binding approximation.
\end{abstract}

\section{INTRODUCTION}

Identification of spectroscopic features and correlation with nanotube geometric structure will aid attempts to purify, separate and sort nanotubes based on their electronic structure. Carbon nanotubes are labeled by two integers $n$ and $m$ that define the circumferential length, $\pi d_{t}$ and chiral angle $\alpha$ of the vector connecting two periodic atom locations as a conceptual graphene sheet is "rolled" into the tube [1]. Advances in the solution phase dispersion and processing of carbon nanotubes [2], and spectrofluormetric detection have lead to a definitive $(\mathrm{n}, \mathrm{m})$ assignment of semiconducting features by exploiting their band-gap fluorescence [3].

Alternatively, metallic nanotubes do not fluoresce, but still posses absorption maxima corresponding to inter-band electronic transitions. Features in the Raman spectrum which are distinct for particular $(n, m)$ nanotubes couple to these transitions as the probing laser energy becomes commensurate with the transition energy [4]. In this way, by looking at the intensity profile of the Raman spectrum through a range of closely spaced excitation energies, these features can be correlated and assigned to (n,m) nanotubes.

Nanotubes produced by $\mathrm{CO}$ disproportionation over Fe generally have smaller diameters $(0.6$ to $1.2 \mathrm{~nm})$ compared to those produced by laser ablation, CVD and electric arc methods and this is ideal for spectroscopic assignment purposes [5-7]. As the 
nanotube diameter increases, the norm of the electronic wave vector becomes small and chirality differences in electronic structure are minimized [8]. Also, the radial breathing mode frequency (RBM) in the Raman spectrum is inversely proportional to nanotube diameter [9], meaning that larger diameter distributions may provide unfavorable resolution between discrete nanotube frequencies. Recent solution phase dispersion methods have demonstrated the ability to produce isolated, individual nanotubes for spectroscopic analysis [2]. In this work, the optical transitions of metallic nanotubes are measured directly using Raman excitation profiles in the range from 627 to $565 \mathrm{~nm}$ and also between 458 to $502 \mathrm{~nm}$ laser excitation. Correlation of the RBM frequency with diameter allows for an unambiguous $(\mathrm{n}, \mathrm{m})$ assignment.

\section{Experimental}

Raman spectra were obtained using sodium dodecyl sulfate suspended nanotubes in aqueous solution prepared as reported previously [2]. The $\mathrm{pH}$ of the sample was adjusted to 10 by the addition of $1 \mathrm{~N} \mathrm{NaOH}$ to fully restore optical transitions that become diminished at lower $\mathrm{pH}$ [10]. Raman spectroscopy was performed by lasing a Coherent 599 dye laser employing a Rhodamine 590 dye pumped using the $488 \mathrm{~nm}$ line of a Lexel 95 water cooled $\mathrm{Ar}^{+}$laser. A liquid $\mathrm{N}_{2}$ cooled CCD array detector was used for detection. Discrete $\mathrm{Ar}^{+}$lines at 457.5, 476.7, 488, 496.9, 502 and $514.5 \mathrm{~nm}$ were also employed. Wavelength and intensity were calibrated using Ne and black body emission lamps respectively.

\section{Radial Breathing Mode/Diameter Relationship}

The graphene plane almost uniquely quantizes nanotube diameters. The frequency of the Raman radial breathing mode is a monotonic function of the diameter and modeled as linear in inverse diameter with an offset $[3,9]$.

$\omega_{R B M}=\frac{c_{1}}{d_{t}}+c_{2}=\frac{\pi c_{1}}{a_{C-C} \sqrt{3\left(n^{2}+n m+m^{2}\right)}}+c_{2}$

Here, $\omega_{\mathrm{RBM}}$ is the mode frequency, $a_{\mathrm{c}-\mathrm{c}}=0.144 \mathrm{~nm}, \mathrm{~d}_{\mathrm{t}}$ is the nanotube diameter $(\mathrm{nm})$ and $\mathrm{c}_{1}$ and $\mathrm{c}_{2}$ are empirically derived parameters. Previously, our work on the assignment of the semiconducting nanotubes [3] has yielded constants $\mathrm{c}_{1}=223.5(\mathrm{~nm} \mathrm{~cm})$ and $\mathrm{c}_{2}=12.5$ $\left(\mathrm{cm}^{-1}\right.$.) Metallic nanotubes for which $|\mathrm{n}-\mathrm{m}|=3 \mathrm{q}$ where $\mathrm{q}$ is an integer are separated in diameter to greater extent than semi-conducting nanotubes. Hence, probing the region between 457 and $627 \mathrm{~nm}$ where the first inter-band transitions of the metallic nanotubes are dominant for HiPco produced material should produce only those discrete mode frequencies predicted by these constants. For example, the $(12,0)$ and $(8,5)$ appear sequentially in a list of metals sorted by diameter and they are separated by $0.051 \mathrm{~nm}(12$ $\mathrm{cm}^{-1}$.) When these frequencies are plotted versus $1 / \mathrm{d}_{\mathrm{t}}$ as in Fig 1 , statistically identical parameters $c_{1}$ and $c_{2}$ are obtained. This provides an additional confirmation of this correlation and a useful mapping of $\omega_{\mathrm{RBM}}$ and $(\mathrm{n}, \mathrm{m}$.) For example, with few exceptions Eq. 1 , can be used to uniquely assign $(n, m)$ indices of metallic RBM features in the Raman spectrum. 
Figure 1: Observed Raman radial breathing mode frequencies assigned to closest $(n, m)$ species versus inverse diameter. The linear relationship is statistically identical to that produced in [3] and agrees with independent diffraction measurements.

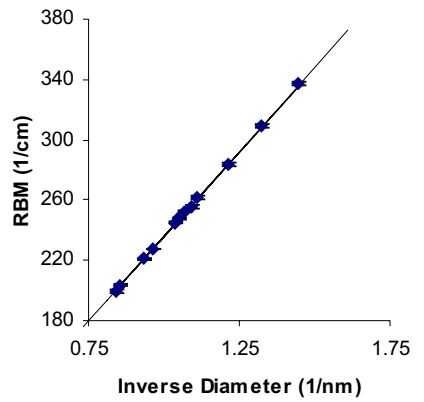

\section{Correlation of Optical Transitions}

Chiral metallic nanotubes $(n \neq m)$ are predicted to demonstrate peak splitting of the $\mathrm{v} 1 \rightarrow \mathrm{c} 1$ transition with positive and negative deviations from an armchair $(\mathrm{n}=\mathrm{m})$ curve $[1$, $8,11]$ due to trigonal warping. We represent the form of this curve (for metallic $\mathrm{v} 1 \rightarrow \mathrm{c} 1$ ) transitions as a truncated asymptotic expansion in $\mathrm{d}_{\mathrm{t}}$ :

$\lambda_{11}^{\text {Armchair }}=\frac{h c}{A} \frac{\S d_{t}}{\text { Cb } a_{c-c}}+\frac{a_{c-c} B}{4 d_{t}}$.

Here, A and B are adjustable parameters. In the simplest single electron model, A is related to the $\mathrm{C}-\mathrm{C}$ interaction energy and equal to the conventional $\gamma_{\mathrm{o}}$ parameter with $\mathrm{B}$ $=1$. The split bands of zig-zag metallic nanotubes can be scaled as positive and negative deviations from eq. 2 such that:

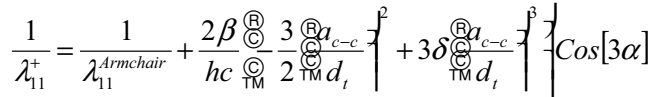

and

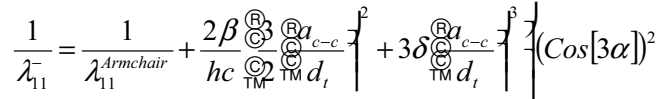

Here, $\alpha$ is the nanotube chiral angle. The latter two parameters $\beta$ and $\delta$ reflect the extent of band splitting and in the simplest example of a single parameter tight binding model are equal to the conventional $\gamma_{0}$ and 1 respectively. In this case $A$ and $B$ are also $\gamma_{0}$ and 1 respectively. Note that in the limit as the norm of the $\mathbf{k}$ vector approaches zero, eq. 3 and 4 approach the familiar limit of $\lambda_{11}=\frac{h c d_{t}}{6 A a_{c-c}}$ with $\mathrm{A}=\gamma_{0}$. The $\operatorname{Cos}[3 \alpha]^{\mathrm{n}}$ factor (n $=1$ for $\lambda^{+}$and 2 for $\lambda^{-}$) scales the armchair deviation for chiral nanotubes. 
We note that the same approach can be used to describe transitions of the semiconducting nanotubes for validation. The armchair curves are scaled by factors of $1 / 3$ and $2 / 3$ for the $\mathrm{v} 1 \rightarrow \mathrm{c} 1$ and $\mathrm{v} 2 \rightarrow \mathrm{c} 2$ semiconductor transitions respectively:

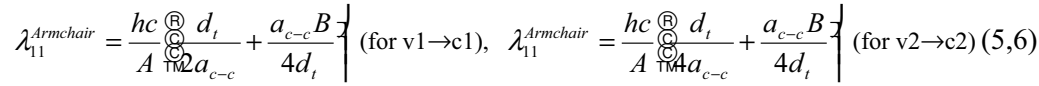

For both sets of transitions:

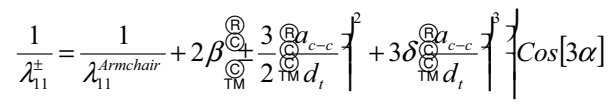

However, the negative root represents the species for which $\bmod 3$ of $(n-m)=1$ with the positive corresponding to $\bmod 3$ of $(\mathrm{n}-\mathrm{m})=2$ for $\mathrm{v} 1 \rightarrow \mathrm{c} 1$. For the $\mathrm{v} 2 \rightarrow \mathrm{c} 2$, the signs are exactly reversed. For comparison, the fitted values for semiconducting transitions are: A $=3.73 \mathrm{eV}, \mathrm{B}=1.63, \beta=1.07 \mathrm{eV}$ and $\delta=-1.62$ for $\mathrm{v} 1 \rightarrow \mathrm{c} 1$ and $\mathrm{A}=3.33 \mathrm{eV}, \mathrm{B}=3.28, \beta$ $=2.77 \mathrm{eV}$ and $\delta=-0.52$ for $\mathrm{v} 2 \rightarrow \mathrm{c} 2$.

\section{Metallic Transitions from Raman Excitation Profiles}

In the Rhodamine $6 \mathrm{G}$ range, four resolvable profiles corresponding to metallic nanotubes are observed and assigned as the $(11,2)$ with $\lambda_{11}=569 \mathrm{~nm},(11,5)$ with $\lambda_{11}=$ $589 \mathrm{~nm}$ and $(12,0)$ with $\lambda_{11}=575 \mathrm{~nm}$ and $(8,8)$ at $\lambda_{11}=589 \mathrm{~nm}$. The $(11,2)$ assignment was selected over the $(7,7)$ despite their identical diameters because of the close proximity of the $(12,0)$ transition. The framework outlined above indicates that the closeness of the transitions suggests similar chiral angles for these two species. Alternatively, the higher energy $\mathrm{Ar}^{+}$laser range monitors primarily two smaller diameter metallic nanotubes $(12,0)$ and $(8,5)$ which demonstrate transitions near $502 \mathrm{~nm}$.

The optimal values of (A, B, $\beta$, and $\delta$ ) for metallic nanotubes were found by nonlinear regression of these observed transitions mapped using the dye laser experiment described above and the two prominent maxima obtained from discrete Ar+ laser energies. These optimal values are: $\mathrm{A}=3.0 \mathrm{eV}, \mathrm{B}=4.47, \beta=2.23 \mathrm{eV}$ and $\delta=-0.78$. The value of $A$ falls within the range of $\gamma_{0}$ parameters reported for optical calculations $[1$, $4,11]$ and suggests that excitonic effects, if operative for metallic transitions, are minor compared to semiconducting nanotubes. Figure 2 compiles the $(n, m)$ assignment and extrapolated transition wavelengths with the observed $(n, m)$ species highlighted. We note that the extrapolated model is able to predict the appearance of weaker metallic RBM features from both scanning experiments. The $(7,4)$ nanotube at $304 \mathrm{~cm}^{-1}$ appears in only the 457 and $476 \mathrm{~nm} \mathrm{Ar}+$ spectra and we note that it has two predicted transitions near these energies. The $(8,2)$ feature at $316.5 \mathrm{~cm}^{-1}$ is apparent in the $502 \mathrm{~nm}$ spectrum with a predicted transition at $491 \mathrm{~nm}$. 

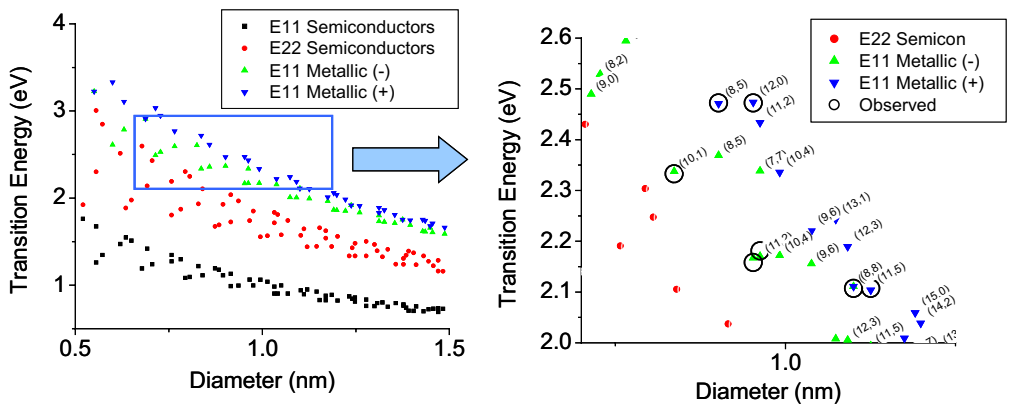

Figure 2: (left) A revised Katura plot from equations (2) through (7) for electronic transitions of single walled carbon nanotubes probed by optical methods. (right) Observed and extrapolated metallic carbon nanotube assignment for the $1^{\text {st }}$ inter-band

\section{Acknowledgements}

This research has been supported by the NSF (CHE-9900417), the NSF Focused Research Group on Fullerene Nanotube Chemistry (DMR-0073046), the NSF Center for Biological and Environmental Nanotechnology (EEC-0118007), and the Robert A Welch Foundation (C-0689 and C-0807). Support from NASA (NCC 9-77) for development of the HiPco method is also gratefully acknowledged.

\section{References}

1. Saito, R., G. Dresselhaus, and M.S. Dresselhaus, Physical Properties of Carbon Nanotubes. 1998, London: Imperial College Press.

2. Strano, M.S., et al., The role of surfactant adsorption during ultra-sonication in the dispersion of single walled carbon nanotubes. J. Nanosci. and Nanotech., 2003. (in press.).

3. Bachilo, S.M., et al., Structure-Assigned Optical Spectra of Single-Walled Carbon Nanotubes. Science, 2002.

4. Dresselhaus, M.S., et al., Raman spectroscopy on isolated single wall carbon nanotubes. Carbon, 2002. 40(12): p. 2043-2061.

5. Thess, A., et al., Crystalline ropes of metallic carbon nanotubes. Science, 1996. 273(5274): p. 483487.

6. Reich, S., C. Thomsen, and P. Ordejon, Electronic band structure of isolated and bundled carbon nanotubes. Physical Review B, 2002. 65(15): p. 155411.

7. Bronikowski, M.J., et al., Gas-phase production of carbon single walled nanotubes from carbon monoxide via the HiPco process: A parametric study. Journal of Vacuum Science \& Technology, 2001.

8. Saito, R., G. Dresselhaus, and M.S. Dresselhaus, Trigonal warping effect of carbon nanotubes. Physical Review B, 2000. 61(4): p. 2981-2990.

9. Sauvajol, J.L., et al., Phonons in single wall carbon nanotube bundles. Carbon, 2002. 40: p. 16971714.

10. Strano, M.S., et al., Reversible, Band-Gap Selective Protonation of Single-Walled Carbon Nanotubes. Journal of Physical Chemistry B, 2002.

11. Reich, S. and C. Thomsen, Chirality dependence of the density-of-states singularities in carbon nanotubes. Physical Review B, 2000. 62(7): p. 4273-4276. 